\title{
Anatomy of Spatial Attention: Insights from Perfusion Imaging and Hemispatial Neglect in Acute Stroke
}

\author{
Argye E. Hillis, ${ }^{1}$ Melissa Newhart, ${ }^{1}$ Jennifer Heidler, ${ }^{1}$ Peter B. Barker, ${ }^{2}$ Edward H. Herskovits, ${ }^{3}$ and \\ Mahaveer Degaonkar ${ }^{1}$ \\ Departments of ${ }^{1}$ Neurology and ${ }^{2}$ Radiology, Johns Hopkins University School of Medicine, Baltimore, Maryland 21287, and ${ }^{3}$ Department of Radiology, \\ University of Pennsylvania School of Medicine, Philadelphia, Pennsylvania 19104-6055
}

The site of lesion responsible for left hemispatial neglect after stroke has been intensely debated recently. Some studies provide evidence that right angular lesions are most likely to cause left neglect, whereas others indicate that right superior temporal lesions are most likely to cause neglect. We examine two potential accounts of the conflicting results: (1) neglect could result from cortical dysfunction beyond the structural lesion in some studies; and (2) different forms of neglect with separate neural correlates have been included in different proportions in separate studies. To evaluate these proposals, we studied 50 patients with acute right subcortical infarcts using tests of hemispatial neglect and magnetic resonance diffusion-weighted and perfusion-weighted imaging performed within $48 \mathrm{~h}$ of onset of symptoms. Left "allocentric" neglect (errors on the left sides of individual stimuli, regardless of location with respect to the viewer) was most strongly associated with hypoperfusion of right superior temporal gyrus (Fisher's exact test; $p<0.0001$ ), whereas left "egocentric" neglect (errors on the left of the viewer) was most strongly associated with hypoperfusion of the right angular gyrus $(p<0.0001)$. Patients without cortical hypoperfusion showed no hemispatial neglect. Because the patients did not have cortical infarcts, our data show that neglect can be caused by hypoperfused dysfunctional tissue not detectable by structural magnetic resonance imaging. Moreover, different forms of neglect were associated with different sites of cortical hypoperfusion. Results help explain conflicting results in the literature and contribute to the understanding of spatial attention and representation in the human brain.

Key words: hemispatial neglect; spatial attention; parietal cortex; temporal cortex; magnetic resonance perfusion-weighted imaging; stroke

\section{Introduction}

The location of brain damage most likely to cause hemispatial neglect has been intensely debated. The critical lesion responsible for neglect was right superior temporal gyrus (STG) in some studies (Karnath et al., 2001, 2004a,b) and right inferior parietal lobule, particularly angular gyrus (AG), in others (Vallar, 2001; Marshall et al., 2002; Mort et al., 2003). Although discrepant results have been ascribed to differences in scan resolution or in the method of lesion mapping (Mort et al., 2004), previous studies implicating right $\mathrm{AG}$ in causing neglect, like recent studies implicating STG, did not use high-resolution magnetic resonance imaging (MRI) or spatial normalization (Brain, 1941; Critchley, 1949; Vallar and Perani, 1986; Heilman et al., 1994; Friedrich et al., 1998).

An alternative explanation of the discordant results is that the proportions of patients with different types of neglect may have varied across studies, because neglect is a heterogenous condition (Heilman et al., 1993, 1997; Chatterjee, 1994; Stone et al., 1998;

Received 0ct. 29, 2004; revised Feb. 14, 2005; accepted Feb. 14, 2005.

This work was supported by National Institutes of Health Grants R01 NS047691 and P41 RR15241.

Correspondence should be addressed to Dr. Argye E. Hillis, Associate Professor of Neurology, Johns Hopkins University School of Medicine, Johns Hopkins Hospital, Phipps 126, 600 North Wolfe Street, Baltimore, MD 21287. E-mail: argye@jhmi.edu.

DOl:10.1523/JNEUROSCI.4468-04.2005

Copyright $\odot 2005$ Society for Neuroscience $\quad 0270-6474 / 05 / 253161-07 \$ 15.00 / 0$
Bisiach and Vallar, 2000; Halligan et al., 2003). Neglect can be distinguished along the lines of input/output dimensions, sectors of space, sensory modality, processing domain, or reference frame, which might have distinct neuroanatomical correlates (Vallar et al., 2003). For example, some patients neglect stimuli on the left side of space, as defined by the midplane of the body, head, or visual field ("egocentric" neglect), whereas others neglect the left side of each individual "stimulus," regardless of its location with respect to the viewer ("allocentric" neglect) (Chatterjee, 1994; Vallar et al., 2003).

Both egocentric and allocentric neglect cause left-sided errors on the cancellation tasks used in previous studies. Patients with allocentric neglect may omit items on the left side of the page because they process the page of items as a single stimulus when attention to both sides of each item is not required. Patients with egocentric neglect may omit items on the left side of the page because these items fall on their left sides. These types of neglect can be distinguished with tasks involving stimuli with left and right targets (e.g., left or right gaps in circles) presented across the page (Ota et al., 2001). Patients with allocentric neglect miss left targets within stimuli on both sides of the page; patients with egocentric neglect detect both left and right targets within stimuli on the right but fail to respond to stimuli on the left side of the page.

A second account of the conflicting results is that deficits in 
acute stroke are caused not only by the infarct demonstrated on structural imaging but also by areas of hypoperfusion beyond the infarct (Barber et al., 1998; Beaulieu et al., 1999). Therefore, neglect in acute stroke might be attributable to hypoperfused regions not visible on structural MRI.

To evaluate these potential explanations of the conflicting results and to investigate neural regions responsible for spatial processing, we identified regions of cortical hypoperfusion associated with egocentric versus allocentric neglect in acute stroke patients with subcortical infarcts. We hypothesized that the site of cortical hypoperfusion varies in allocentric versus egocentric neglect.

\section{Materials and Methods}

Subjects. A consecutive series of 50 consenting right-handed patients with acute ischemic infarct confined to right subcortical areas (basal ganglia, thalamus, and/or surrounding white matter) were studied within $48 \mathrm{~h}$ of onset of stroke symptoms. Exclusion criteria included the following: altered level of consciousness, ongoing sedation, hemorrhage, contraindication to MRI, or signal abnormality in the cortex on any of the MRI sequences. Patients with hemianopia were not excluded, because severe neglect can mimic hemianopia (Vallar et al., 1991), although hemianopia does not mimic neglect. All patients provided informed consent for the study, using methods and consent forms approved by the Johns Hopkins Institutional Review Board. Age ranged from 35 to 85 years (mean, $61.7 \pm 13.4$ ). Education ranged from 5 to 16 years (mean, $11.5 \pm 3.2$ ).

Imaging. All patients had the following MRI sequences (the Johns Hopkins Acute Stroke Protocol) within 48 h of onset of symptoms: axial T2, fluid-attenuated inversion recovery, gradient echo, diffusionweighted images (DWIs), apparent diffusion coefficient (ADC) maps, dynamic contrast perfusion-weighted images (PWIs), and magnetic resonance angiogram of the circle of Willis. The reported analyses used DWIs (after confirming the acuity of the lesion as dark on ADC maps) and PWIs (coregistered to T2 to provide anatomical boundaries that are less visible on PWIs). DWI and PWI scans were $5 \mathrm{~mm}$ in thickness and provided whole-brain coverage. Areas of hypoperfusion on PWIs were determined with time-to-peak maps, using Scion (Frederick, MD) Image software. Two trained technicians, blinded to the results of neglect testing, examined 20 regions of interest (ROIs) for the presence or absence of hypoperfusion. Hypoperfusion was defined as $>2.5 \mathrm{~s}$ mean delay in time-to-peak arrival of contrast across voxels in the ROI relative to the homologous region in the left hemisphere. This threshold was based on evidence that tissue with this degree of hypoperfusion is dysfunctional, although it may not be at risk for progressing to infarction, whereas a delay of $<2.5 \mathrm{~s}$ is not associated with dysfunction (Hillis et al., 2000, 2001). ROIs were defined manually using the Damasio and Damasio (1989) templates. The 20 ROIs were the insula and Brodmann's areas (BAs) 4, 5, 6, 7, 9, 10, 11, 18, 19, 20, 21, 22 (superior temporal gyrus), 37, 38, 39 (angular gyrus), 40 (supramarginal gyrus), 44, 45, and 46 in the right hemisphere. These ROIs were chosen because they have been reported previously to be infarcted in patients with hemispatial neglect (Heilman et al., 1994, 1997) or have been reported to show activation in functional imaging studies of spatial attention (for review, see Vallar et al., 2003). Interjudge reliability in identifying the presence or absence of hypoperfusion as defined above in each ROI across the two technicians was very high ( $96 \%$ point-to-point agreement).

PWI scans, coregistered with T2, have high spatial resolution, in that it is possible to analyze the degree of delay in the arrival of contrast in each voxel of the image. However, we did not use a voxel-based approach to structure-function mapping, because a very large number of subjects both with and without hypoperfusion of each voxel are required to con- duct such an analysis and still avoid identifying random associations by applying Bonferonni's correction for multiple comparisons. It is also recognized that defining ROIs by Brodmann's areas has limitations (e.g., individual variability in the cytoarchitectural fields); however, this method has high interjudge reliability, some theoretical rationale, and some empirical support (because lesions involving specific Brodmann's areas have been associated with hemispatial neglect). For these reasons, our use of Brodmann's area landmarks should be considered approximate. Although large areas of brain often showed small changes in perfusion (1-2 s delay relative to the homologous region in the intact hemisphere) on PWIs, we used a threshold of delay that has been demonstrated previously to correspond to dysfunction.

Neglect testing. Tests of hemispatial neglect included the following: copying a scene of two trees, a house, and a fence (Ogden, 1985); modifications of the line cancellation test (Albert, 1973); line bisection, in which the page was presented $45^{\circ}$ to the left and $45^{\circ}$ to the right of the midsagittal plane and at the midsagittal plane of the viewer (10-12 inches from the trunk); reading sentences; and a gap-detection test (Ota et al., 2001). In the gap-detection test, a page of 30 circles was presented: 10 circles had a gap on the left side, 10 had a gap on the right side, and 10 had no gap. The subject was instructed to circle each complete circle and place an $\mathrm{X}$ on each circle with a gap. Two forms of this test were presented: one with large circles and one with small circles.

Scoring of each test included percentage of error responses on each test (or deviation from midpoint in line bisection measured as percentage of line length). In copying a scene, each stroke in the scene to be copied was counted as a point; each omitted stroke was scored as one error, and each distorted or misplaced stroke was scored as one-half of an error. In the gap-detection test, there were three percentage-of-error scores: percentage of circles omitted (no response to the stimulus), percentage of left gaps missed, and percentage of right gaps missed, among the stimuli for which there was some response (an error consisted of incorrectly circling a circle with a gap). No normal subject made $>10 \%$ errors on any of these tests (Hillis et al., 2000, 2002); therefore, neglect was defined as $>10 \%$ errors on the task, with a simple majority of errors on the left. For line bisection, neglect was defined as deviation to the right by $>10 \%$ of line length. In addition, each subject was evaluated with regard to the presence of allocentric neglect and/or egocentric neglect. Criteria for allocentric neglect included the following: (1) significantly more errors on the left than the right (by Fisher's exact test) or $>10 \%$ errors on the left and no errors on the right side of individual items in the scene on both sides of the page in the scene-copying task; (2) significantly more errors on the left than the right or $>10 \%$ errors on the left and none on the right sides of individual words on both sides of sentences in the oral sentence reading test; and/or (3) significantly more errors on left gaps than right gaps or $>10 \%$ errors on left gaps and no errors on right gaps in circles on both sides of the page in the Ota et al. (2001) task (Fig. 1). Criteria for egocentric neglect included the following: (1) significantly (by Fisher's exact test) more errors on the left than right side of the scene or $>10 \%$ errors on the left and no errors to the right of the leftmost response (e.g., 
Table 1. Relationship between each type of neglect (or no neglect) and location of subcortical infarct

\begin{tabular}{lllll}
\hline & No & $\begin{array}{l}\text { Egocentric } \\
\text { neglect } \\
\text { only }\end{array}$ & $\begin{array}{l}\text { Allocentric } \\
\text { neglect only }\end{array}$ & $\begin{array}{l}\text { Egocentric plus } \\
\text { allocentric neglect }\end{array}$ \\
\hline Thalamus & 16 & 1 & 0 & 0 \\
Caudate & 5 & 4 & 2 & 0 \\
Putamen & 9 & 6 & 2 & 0 \\
Globus pallidus & 7 & 5 & 0 & 0 \\
Internal capsule & 9 & 3 & 0 & 0 \\
White matter & 9 & 6 & 1 & 1 \\
\hline
\end{tabular}

Values are given in number of patients among the total subject pool of 50.

omission of whole figures on the left) in the scene-copying test; (2) significantly more omitted words on the left than the right sides of sentences or $>10 \%$ omissions on the left and none on the right of the leftmost response in oral sentence reading; and/or (3) significantly more omissions of stimuli on the left than right side of the page or $>10 \%$ omissions of stimuli on the left side and no omissions on the right of the leftmost response in the Ota et al. (2001) task.

Statistical analysis. Associations between each ROI and each type of neglect (allocentric and egocentric) were evaluated with Fisher's exact analysis. An $\alpha$ level of $p<0.00125$ was used, based on Bonferroni's correction for multiple comparisons (20 ROIs $\times$ two types of neglect). In addition, stepwise linear regression analyses were also performed to determine regions of cortical hypoperfusion and regions of subcortical infarct that contributed to predicting each type of neglect.

\section{Results}

All 16 subjects who met our criteria for either form of hemispatial neglect had cortical hypoperfusion in the right middle cerebral artery or posterior cerebral artery territory, consistent with previous results (Vallar et al., 1988; Hillis et al., 2002). The one patient with cortical hypoperfusion, but without neglect, had hypoperfusion in right BA 37. There was a very strong association between hemispatial neglect (of either type) and cortical hypoperfusion $(p<0.0001)$. There were no significant associations by Fisher's exact test between the site of subcortical infarct and the presence of neglect or type of neglect (Table 1). Some patients had infarct involving more than one subcortical region and are therefore included more than once in Table 1.

Among the 16 patients with neglect, 4 had exclusively allocentric neglect, 11 had exclusively egocentric neglect, and 1 had both types of neglect. Allocentric neglect (total, $n=5$ ) was significantly associated only with hypoperfusion of right BA 22 (superior temporal gyrus; $p<0.0001$ ) and hypoperfusion of right BA 37 (posterior inferior temporal gyrus; $p<0.0001$ ). Examples of patients with hypoperfusion of right superior temporal gyrus and allocentric neglect are shown in Figure 2.

In contrast, egocentric neglect (total, $n=12$ ) was most strongly associated with hypoperfusion of right BA 39 (angular gyrus; $p<0.0001$ ) and BA 40 (supramarginal gyrus; $p<0.0001$ ) but was also significantly associated with hypoperfusion of right BA 44 (posterior inferior frontal gyrus; $p<0.0002$ ) and right BA 19 (visual association cortex; $p<0.0002$ ). We could not demonstrate a significant association between egocentric neglect and hypoperfusion of right BA 22 (superior temporal gyrus; $p=0.38$ ) or BA 37 (posterior inferior temporal gyrus; $p=0.073$ ) as we had for patients with allocentric neglect. Examples of patients with hypoperfusion of right posterior inferior frontal gyrus and/or the right inferior parietal lobule (angular and supramarginal gyri) and egocentric neglect are shown in Figures 3 and 4.

The area of hypoperfusion most strongly associated with egocentric neglect by $\chi^{2}$ tests was BA 39 (angular gyrus; $\chi^{2}=35.4$; $p<0.0001)$; the area of hypoperfusion most strongly associated with allocentric neglect was BA 22 (superior temporal gyrus; $\chi^{2}=24.1 ; p<0.0001$ ). The numbers of patients with hypoperfusion in these areas, with each type of neglect or with no neglect, are shown in Table 2.

A stepwise linear regression analysis, using $p=0.002$ for inclusion in the model (to correct for multiple comparisons), was performed to identify the combination of areas of cortical hypoperfusion and areas of subcortical infarct (a total of 26 anatomic regions) that predicted the continuous variable egocentric neglect. The best regression model $(r=0.86$; $p<0.001)$ was characterized by the following equation, in which HP refers to hypoperfusion of a region, a binary variable for which 1 represents hypoperfusion, and 0 represents normal perfusion: egocentric neglect $=0.92 \times \mathrm{HP} \mathrm{BA} 39+0.73 \times \mathrm{HP} \mathrm{BA} 44-0.62 \times \mathrm{HP}$ insula +0.018 .

Note that hypoperfusion of the insula negatively predicted viewer-center neglect and that none of the subcortical areas of infarct predicted egocentric neglect. Hypoperfusion of BA 40 did not independently predict egocentric neglect. It may have shown a significant association with egocentric neglect by Fisher's exact test, because it is frequently hypoperfused when angular gyrus is hypoperfused.

Similarly, to identify the combination of areas that predicted allocentric neglect, a stepwise linear regression analysis was performed, using $p=0.002$ for inclusion in the model. The regression model $(r=0.71 ; p<0.001)$ included only BA 22: allocentric neglect $=1.0 \times$ HP BA $22+0$ (hypoperfusion of BA 22 was the only significant predictor of allocentric neglect).

To confirm our findings that regional cortical hypoperfusion predicted egocentric versus allocentric neglect, rather than neglect on a particular test, we also performed a similar analysis of the areas of cortical hypoperfusion or subcortical infarct associated with scores on each neglect test. Stepwise linear regression analysis, using $p=0.002$ for inclusion in the model for each test, yielded no areas of abnormality that predicted performance on any single test. There were also no significant associations by Fisher's exact test between neglect on a particular test (collapsing across type of neglect shown on the test) and hypoperfusion of any of the ROIs.

Performance on line bisection was not included among criteria for each type of neglect, because a deviation in line bisection to the ipsilesional side may reflect either egocentric or allocentric neglect (or extinction of the left segment) (Riestra et al., 2001). A greater deviation to the ipsilesional (right) side when the line is presented on the viewer's left side would reflect egocentric neglect, but this effect might not occur with egocentric neglect defined by head-centered or eye-centered coordinates (depending on whether the patient turned his head or eyes to the left, which is difficult to control). Of the 12 patients with egocentric neglect, seven $(58 \%)$ showed a greater deviation of at least $1 \mathrm{~cm}$ to the right (worse left neglect) in line bisection when the line was placed on the left side of the viewer. This result indicates that the left neglect in these seven patients was defined at least by location with respect the midsagittal plane of the trunk, assuming that the patients turned their heads and eyes toward the stimulus. Of the five patients with allocentric neglect, only one showed a greater deviation of at least $1 \mathrm{~cm}$ in line bisection when the line was on the left side of the trunk; this patient had both egocentric and allocentric neglect by our previously stated criteria.

Of the 13 patients with hypoperfusion of BA 39 and/or 40 (inferior parietal lobule), four also had hypoperfusion of BA 22. Of the seven patients with hypoperfusion of BA 22, four also had 
hypoperfusion of BA 39 and/or 40. Thus, we might have expected up to four patients to show both types of neglect. However, only two patients had hypoperfusion of both BA 39 and 22. One was the only patient who met criteria for both egocentric and allocentric neglect. This patient had hypoperfusion of right BA 22, 37, 39, 40, 44,4 , and 6 . Previous studies of series of eight or more patients with neglect that have distinguished between egocentric and allocentric (stimulus/object-centered) neglect have found similar distributions of types of neglect, with egocentric neglect being the most common, allocentric neglect less common, and both types of neglect being the least common (Chatterjee, 1994; Hillis and Rapp, 1998; Marsh and Hillis, 2005). However, most reports of dissociations between these types of neglect have been single case studies or studies of a pair of patients with contrasting patterns of performance (Driver and Halligan, 1991; Arguin and Bub, 1993; Ota et al., 2001). Although several authors have proposed an interaction between frames of reference of neglect (Rapcsak et al., 1987; for review, see Behrmann and Geng, 2002), our study and previous studies demonstrate a clear dissociation using the same tasks and instructions.

Although severe neglect can mimic homonymous hemianopia (because neglect can cause a failure to respond to stimuli presented on the contralesional side), field cuts do not typically mimic neglect, because eye movements can easily compensate. Nevertheless, to confirm that our results could not be explained by the presence of a field cut, we evaluated the relationship between each type of neglect and contralesional field cuts (as tested by confrontation), using the Fisher's exact test with an $\alpha$ level of $p<0.05$. We could not demonstrate a significant association between either type of neglect and any of the following: complete left homonymous hemianopia (with or without macular sparing), left upper quadrantanopia, or left lower quadrantanopia. Of the 12 patients with egocentric neglect, two had hemianopia and none had quadrantanopia. Of the five patients with allocentric neglect, two had hemianopia, one had left lower quadrantanopia, and none had left upper quadrantanopia. Of the 34 patients with no neglect, three had left hemianopia, two had left lower quadrantanopia, and none had left upper quadrantanopia.

\section{Discussion}

The results reported here demonstrate that allocentric and egocentric neglect can be attributable to dysfunction of different cortical regions and that hemispatial neglect in acute stroke can be associated with hypoperfusion of noninfarcted regions. The cortical hypoperfusion rather than the subcortical infarct likely caused the neglect because (1) there was no significant association between the site of subcortical lesion and the presence or type of neglect and (2) reperfusion of the cortex resulted in recovery of function despite the continued presence of the subcortical infarct in previous studies (Hillis et al., 2002, 2003a,b). This study was not meant to be the definitive study on the anatomy of
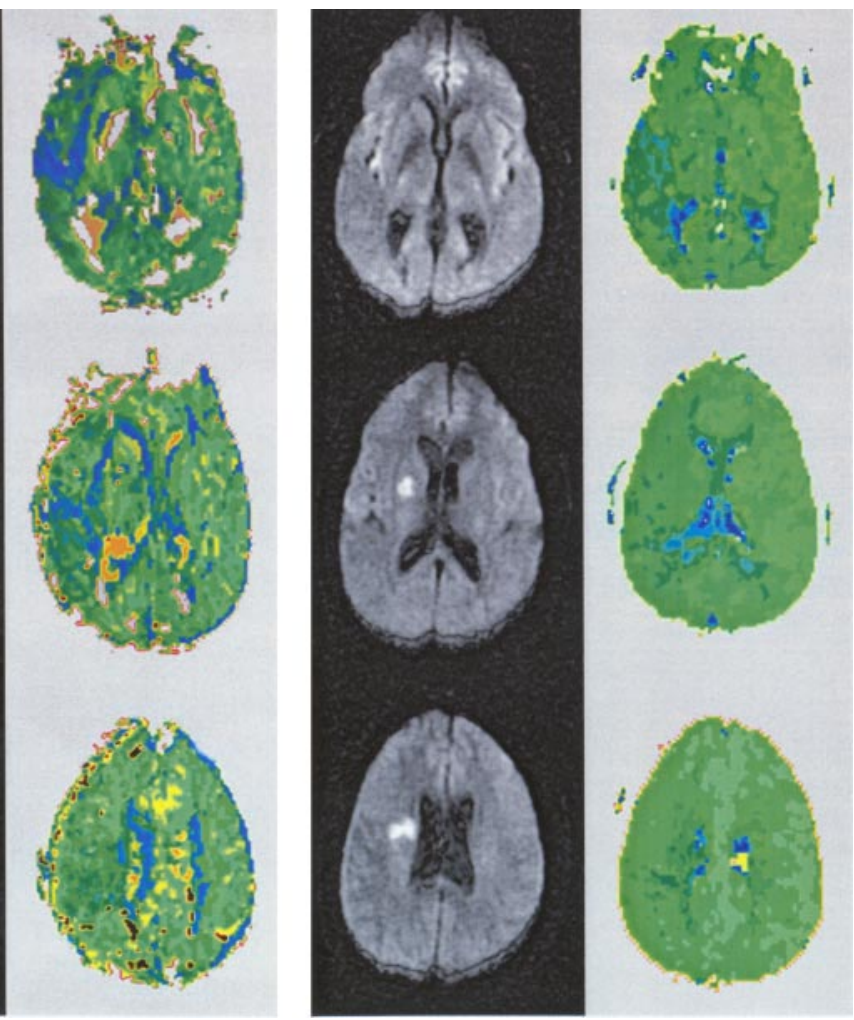

Figure 2. DWI (columns 1 and 3) and PWI (columns 2 and 4) scans of two patients with allocentric neglect and hypoperfusion of right posterior superior temporal cortex (but not right angular gyrus or inferior frontal gyrus). In this and subsequent figures, regions of hypoperfusion appear blue.

neglect but to demonstrate the importance of refining the question. Different right-hemisphere lesions may each disrupt computation or processing of the left side of spatial representations used to direct movements or recognize stimuli, because the undamaged left-hemisphere homolog in each case may be biased toward the right.

Although some investigators (McGlinchey-Berroth et al., 1996) have been unable to identify neuroanatomical subtypes of neglect, Vallar et al. (2003) reviewed evidence for distinct neuroanatomical correlates of different types of neglect distinguished by dimension, sector of space, and task (Mesulam, 1999). Vallar et al. (2003) also reported evidence that allocentric and egocentric neglect can dissociate, noting that egocentric spatial representations might be necessary for organizing goal-directed movements, whereas allocentric representations might be necessary for object identification. They reviewed functional imaging studies that converge in support of the hypothesis that visuomotor transformation required for reaching in peripersonal space engages premotor and "dorsal” parietal regions, whereas locations outside of peripersonal space and more object-like stimuli engage more "ventral" regions. However, previous studies have not provided evidence that distinct lesions in the dorsal or ventral streams result in egocentric versus allocentric neglect. One positron emission tomography study showed that both "spacebased" and "object-based" attention result in activation of the posterior parietal and prefrontal cortices, but their object-based task required attending to the location of one object relative to another, as defined by left versus right of the viewer (Fink et al., 1997). Other functional imaging studies have demonstrated that mapping contralateral space in retinotopic coordinates is associated with parietal activation (Sereno et al., 2001). 

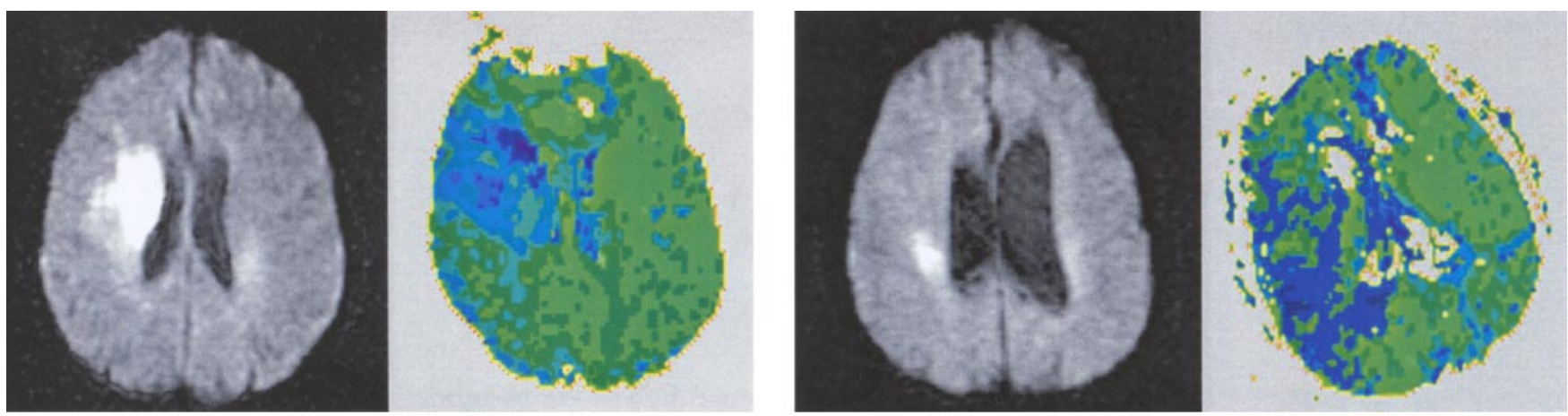

Figure 3. DWI (columns 1 and 3) and PWI (columns 2 and 4) scans of patients with egocentric neglect. Left, Egocentric neglect associated with right posterior inferior frontal gyrus hypoperfusion. Right, Egocentric neglect associated with right inferior frontal gyrus, supramarginal gyrus, and angular gyrus hypoperfusion.
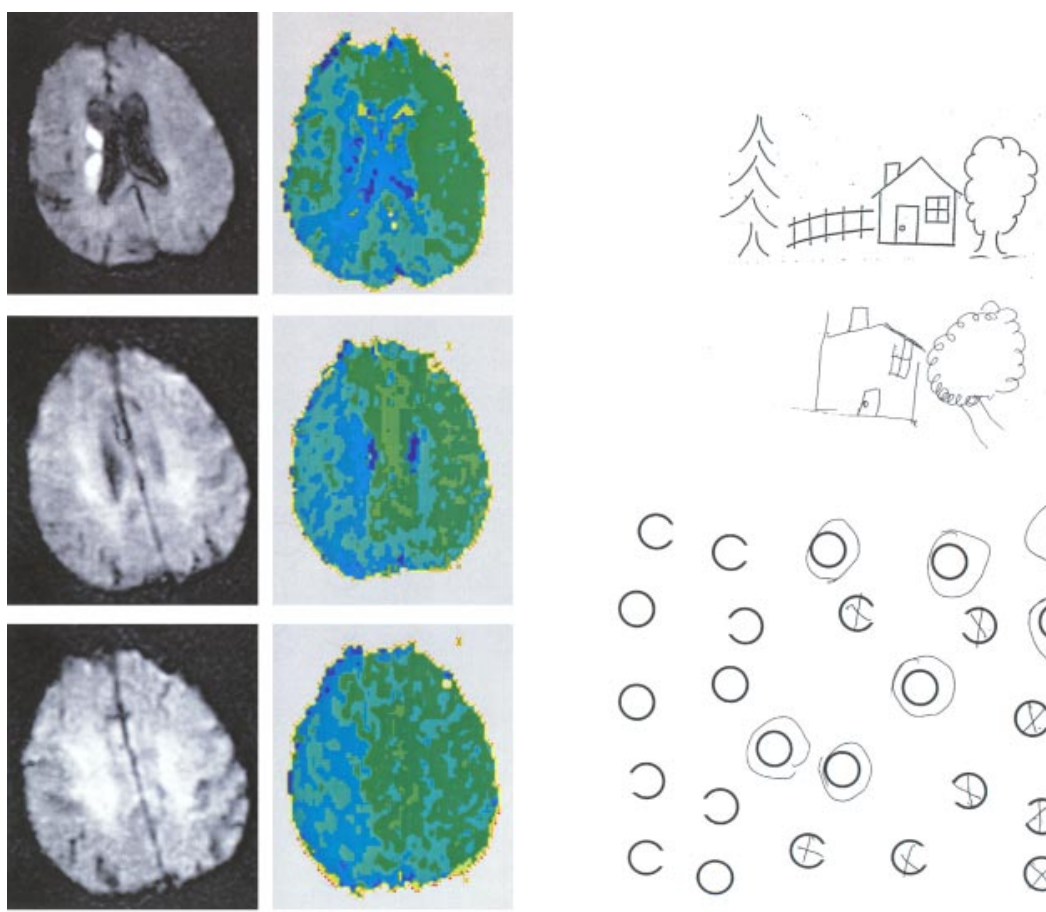

Figure 4. Scans and test results of a patient with egocentric neglect associated with right inferior frontal gyrus, supramarginal gyrus, and angular gyrus hypoperfusion.

Table 2. Relationship between each type of neglect (or no neglect) and hypoperfusion of BA 22 and 39

\begin{tabular}{lccll}
\hline & $\begin{array}{l}\text { No neglect } \\
(n=34)\end{array}$ & $\begin{array}{l}\text { Egocentric } \\
(n=11)\end{array}$ & $\begin{array}{l}\text { Allocentric } \\
\text { neglect only } \\
(n=4)\end{array}$ & $\begin{array}{l}\text { Egocentric plus } \\
\text { allocentric } \\
\text { neglect }(n=1)\end{array}$ \\
\hline BA 22 (STG) & 1 & 1 & 4 & 1 \\
BA 39 (angular gyrus) & 1 & 10 & 1 & 1 \\
Neither 22 nor 39 & 32 & 0 & 0 & 0 \\
\hline
\end{tabular}

Values are given in number of patients among the total subject pool of 50.

We distinguished between egocentric and allocentric neglect and found these to be associated with distinct regions of neural dysfunction. Generally consistent with the conclusions of Vallar et al. (2003), we found that egocentric neglect was associated with frontal and dorsal hypoperfusion in right posterior inferior frontal gyrus, angular gyrus, and supramarginal gyrus (BA 44, 39, and 40) and visual association cortex (BA 19), whereas allocentric neglect was associated with more ventral hypoperfusion, including right superior temporal gyrus (BA 22) and posterior inferior temporal gyrus (BA 37). These results would account for the finding reported by Karnath et al. (2001, 2004a) that superior temporal lesions cause neglect, if these studies included a preponderance of patients with allocentric neglect. As noted, their tests of neglect did not distinguish egocentric and allocentric frames of reference.

The areas identified as responsible for neglect also have connections that we would expect to allow multimodal spatial attention. BA 22 and BA 39/40 in humans are directly connected via white-matter tracts and both (directly or indirectly) receive auditory input from Heschel's gyrus, visual information from inferotemporal cortex, and somatosensory projections from the medial and superior parietal cortices and project to premotor and motor cortices (Nieuwenhuys et al., 1988; Catani et al., 2005). BA 39/40 is probably the human correlate of superior temporal sulcus in primates, the area in which lesions reliably cause egocentric neglect in primates (Watson et al., 1994) and in which single fibers show polymodal receptive fields and code locations of objects with respect to the viewer (Bruce et al., 1981; Baker et al., 2000).

Our findings are compatible with the proposal that dorsalstream processing requires computation of viewer-centered (eyecentered, head-centered, and trunk-centered) spatial maps for guiding limb and eye movements in space, whereas ventralstream processing requires computation of stimulus- or objectcentered representations for object identification (or withinobjects coding) (Humphreys, 1998). Psychophysical studies in primates indicate that parietal neurons respond to particular shapes in particular orientations, probably because the intended action toward the stimulus (e.g., reaching and grasping) is dependent not only on the location of the object with respect to the viewer and the shape of the object but also on its orientation and size (for review, see Kalaska et al., 2003; Sakata, 2003). In fact, these single-cell recording studies indicate that, instead of a single viewer-centered representation computed in the parietal lobe to direct actions, a series of representations with different viewerdefined reference frames is computed, progressing from retinal and eye-centered coordinates to limb- or even muscle-centered 
coordinates (Andersen and Buneo, 2003; Kalaska et al., 2003). Some neurons have response fields that are defined by both eyeand limb-centered representations or eye- and head-centered representations, indicating that they encode spatial location in both coordinate frames (Andersen and Buneo, 2002, 2003). The coordinate transformations from sensory input to motor output are being intensely investigated, but the construction of these viewer-centered representations for sensory guidance of behavior and saccades is generally ascribed to posterior parietal cortex (in the dorsal stream) (Kalaska et al., 2003). Such maps specify the location of salience or salient stimuli with respect to the viewer; the receptive field of each neuron is characterized by the part of the visual field that is associated with the output vector of that neuron (Bisley and Goldberg, 2003). In contrast, responses of temporal lobe neurons are relatively independent of orientation, size, and location (Gross et al., 1972; Desimone et al., 1984), as predicted if allocentric representations used to identify objects are computed in the temporal lobes.

There exists some controversy regarding the precise neuroanatomy of the ventral and dorsal streams. Some authors have proposed that superior temporal gyrus and inferior parietal lobule have neither strictly dorsal-stream nor strictly ventral-stream roles in visual processing (Shapiro et al., 2002) or that dorsal and ventral streams converge in superior temporal sulcus in primates (an area comparable with BA 39/40 in humans) (Young, 1992). However, our study provides some novel evidence that right superior temporal gyrus and inferior parietal lobule may have distinct roles in allocentric versus egocentric spatial representations in humans.

Egocentric and allocentric variants of neglect might be further subdivided into anatomically relevant subgroups. For example, our tasks confound various potential types of egocentric neglect, such as body-centered, head-centered, and retinocentric neglect (Hillis and Caramazza, 1995). However, dissociations might not occur if neurons responsive to distinct viewer-defined reference frames are intermixed or in close proximity (e.g., within posterior parietal cortex) or if parietal neurons encode spatial location in multiple reference frames (Andersen and Buneo, 2002, 2003).

Our tasks also confound directional hypokinesia (reluctance to move the limb toward the contralesional side) and attentional neglect (reduced attention to the contralesional side). The possibility that these types of neglect have different neural correlates is supported by the observation that they can be dissociated after focal brain damage. Furthermore, there has been some evidence from functional imaging for more frontal (premotor) and basal ganglia involvement in guiding movements in space and more posterior parietal involvement in attentional/perceptual aspects of spatial processing (Vallar et al., 2003). Therefore, the association we identified between egocentric neglect and posterior inferior frontal cortex (BA 44) may reflect the association between directional hypokinesia and posterior frontal regions, whereas the association between egocentric neglect and inferior parietal lobule (BA 39 and 40) may reflect the association between egocentric attentional neglect and posterior parietal cortex. Vallar (2001) reviewed evidence for the association between right frontal lesions and premotor aspects of neglect and between parietal lesions and perceptual aspects of neglect. However, a recent study of six neglect patients did not confirm this proposal (Husain et al., 2000). Three patients with right inferior frontal lesions did not show directional motor biases; instead, three patients with inferior parietal lesions showed both directional motor and perceptual biases. However, the patients with frontal lesions were studied 2 months after stroke onset; any directional motor bias caused by the frontal lesion may have resolved.

Together, our study and other recent studies indicate that hemispatial neglect is a heterogenous syndrome (Hillis and Caramazza, 1995; Hillis and Rapp, 1998; Stone et al., 1998; Mesulam, 1999; Marshall et al., 2002) [for discussion and a model of the components of spatial processing in object recognition, see Halligan et al. (2003)]. Future studies of the anatomy of neglect may be most fruitful if the status of each spatially specific cognitive process is better characterized and the associations between precisely characterized deficits and damage/dysfunction of each neural region are determined. Our work illustrates that studying patients acutely after brain lesions, before reorganization or recovery, and at the same time as structural and perfusion imaging, may complement functional imaging studies in normal subjects and more traditional lesion studies in identifying neural regions critical for computing various spatial representations for locating, identifying, and responding to objects.

\section{References}

Albert ML (1973) A simple test of visual neglect. Neurology 23:658-664.

Andersen RA, Buneo CA (2002) Intentional maps in posterior parietal cortex. Annu Rev Neurosci 25:189-220.

Andersen RA, Buneo CA (2003) Sensorimotor integration in posterior parietal cortex. In: Advances in neurology, Vol 93, The parietal lobes (Siegel AM, Andersen RA, Freund H-J, Spencer DD, eds), pp 159-177. London: Lippincott Williams and Wilkins.

Arguin M, Bub DN (1993) Evidence for an independent stimulus-centered spatial reference frame from a case of visual hemineglect. Cortex 29:349-357.

Baker CI, Keysers C, Jellema T, Wicker B, Perrett DI (2000) Coding of spatial position in the superior temporal sulcus of the macaque. Curr Psychol Lett Behav Brain Cogn 1:71-87.

Barber PA, Darby DG, Desmond PM, Yang Q, Gerraty RP, Jolley D, Donnan GA, Tress BM, Davis SM (1998) Prediction of stroke outcome with echoplanar perfusion- and diffusion-weighted MRI. Neurology 51:418-426.

Beaulieu C, de Crespigny A, Tong DC, Moseley ME, Albers GW, Marks MP (1999) Longitudinal magnetic resonance imaging study of perfusion and diffusion in stroke: evolution of lesion volume and correlation with clinical outcome. Ann Neurol 46:568-578.

Bisiach E, Vallar G (2000) Unilateral neglect in humans. In: Handbook of neuropsychology, Ed 2 (Boller F, Graffman J, eds), pp 195-222. Amsterdam: Elsevier Science.

Bisley JW, Goldberg ME (2003) The role of parietal cortex in the neural processing of saccadic eye movements. In: Advances in neurology, Vol 93, The parietal lobes (Siegel AM, Andersen RA, Freund H-J, Spencer DD, eds), pp 141-157. London: Lippincott Williams and Wilkins.

Brain WR (1941) Visual disorientation with special reference to lesions of the right cerebral hemisphere. Brain 64:244-272.

Bruce C, Desimone R, Gross CG (1981) Visual properties of neurons in a polysensory area in superior temporal sulcus of the macaque. J Neurophysiol 46:369-384.

Catani M, Jones DK, ffytche DH (2005) Perisylvian language networks of the human brain. Ann Neurol 57:8-16.

Chatterjee A (1994) Picturing unilateral spatial neglect: viewer versus object-centred reference frames. J Neurol Neurosurg Psychiatry 57:1236-1240.

Critchley M (1949) The phenomenon of tactile inattention with special reference to parietal lesions. Brain 72:538-561.

Damasio H, Damasio A (1989) Lesion analysis in neuropsychology. New York: Oxford UP.

Desimone R, Albright TD, Gross CG, Bruce C (1984) Stimulus selective properties of inferior temporal neurons in the macaque. J Neurosci 4:2051-2062.

Driver J, Halligan PW (1991) Can visual neglect operate in object-centered coordinates. An affirmative single case study. Cogn Neuropsychol 8:475-496.

Fink GR, Dolan RJ, Halligan PW, Marshall JC, Frith CD (1997) Space-based 
and object-based visual attention: shared and specific neural domains. Brain 120:2013-2028.

Friedrich FJ, Egly R, Rafal RD (1998) Spatial attention deficits in humans: a comparison of superior parietal and temporal-parietal junction lesions. Neuropsychology 12:193-207.

Gross CG, Rocha-Miranda CE, Bender DB (1972) Visual properties of neurons in inferotemporal cortex of the macaque. J Neurophysiol 35:96-111.

Halligan PW, Fink GR, Marshall JC, Vallar G (2003) Spatial cognition: evidence from visual neglect. Trends Cogn Sci 7:125-133.

Heilman KM, Watson RT, Valenstein E (1993) Neglect and related disorders. In: Clinical neuropsychology, Ed 2 (Heilman KM, Valenstein E, eds), pp 279-336. New York: Oxford UP.

Heilman KM, Watson RT, Valenstein E (1994) Localization of lesions in neglect and related disorders. In: Localization and neuroimaging in neuropsychology (Kertesz A, ed), pp 495-524. San Diego: Academic.

Heilman KM, Watson RT, Valenstein E (1997) Neglect: clinical and anatomic aspects. In: Behavioral neurology and neuropsychology (Feinberg TE, Farah MJ, eds), pp 309-318. New York: McGraw-Hill.

Hillis AE, Caramazza A (1995) A framework for interpreting distinct patterns of hemispatial neglect. Neurocase 1:189-207.

Hillis AE, Rapp BC (1998) Unilateral spatial neglect in dissociable reference frames: a comment on Farah, Brunn, Wallace, Wong and Carpenter (1990). Neuropsychologia 36:1257-1262.

Hillis AE, Barker PB, Beauchamp NJ, Gordon B, Wityk RJ (2000) MR perfusion imaging reveals regions of hypoperfusion associated with aphasia and neglect. Neurology 55:782-788.

Hillis AE, Wityk RJ, Tuffiash E, Beauchamp NJ, Jacobs MA, Barker PB, Selnes OA (2001) Hypoperfusion of Wernicke's area predicts severity of semantic deficit in acute stroke. Ann Neurol 50:561-566.

Hillis AE, Wityk RJ, Barker PB, Beauchamp NJ, Gailloud P, Murphy K, Murphy K, Cooper O, Metter EJ (2002) Subcortical aphasia and neglect in acute stroke: the role of cortical hypoperfusion. Brain 125:1094-1104.

Hillis AE, Wityk R, Barker PB, Caramazza A (2003a) Neural regions essential for writing verbs. Nat Neurosci 6:19-20.

Hillis AE, Wityk RJ, Barker PB, Ulatowski JA, Jacobs MA (2003b) Change in perfusion in acute nondominant hemisphere stroke may be better estimated by tests of hemispatial neglect than by the NIHSS. Stroke 34:2392-2398.

Humphreys GW (1998) Neural representation of objects in space: a dual coding account. Philos Trans R Soc Lond B Biol Sci 353:1341-1351.

Husain M, Mattingley JB, Rorden C, Kennard C, Driver J (2000) Distinguishing sensory and motor biases in parietal and frontal neglect. Brain 123:1643-1659.

Kalaska JF, Cisek P, Gosselin-Kessiby N (2003) Mechanisms of selection and guidance of reaching movements in the parietal lobe. In: Advances in neurology, Vol 93, The parietal lobes (Siegel AM, Andersen RA, Freund H-J, Spencer DD, eds), pp 97-119. London: Lippincott Williams and Wilkins.

Karnath HO, Ferber S, Himmelbach M (2001) Spatial awareness is a function of the temporal not the posterior parietal lobe. Nature 411:950-953.

Karnath HO, Fruhmann Berger M, Zopf R, Küker W (2004a) Using SPM normalization for lesion analysis in spatial neglect. Brain 127:E10.

Karnath HO, Fruhmann Berger M, Kuker W, Rorden C (2004b) The anatomy of spatial neglect based on voxelwise statistical analysis: a study of 140 patients. Cereb Cortex 14:1164-1172.

Marsh EB, Hillis AE (2005) Egocentric and allocentric visuospatial and tactile neglect in stroke patients. Stroke, in press.
Marshall JC, Fink GR, Halligan PW, Vallar G (2002) Spatial awareness: a function of the posterior parietal lobe? Cortex 38:253-257.

McGlinchey-Berroth R, Bullis DP, Milberg WP, Verfaellie M, Alexander M, D'Esposito M (1996) Assessment of neglect reveals dissociable behavioral but not neuroanatomical subtypes. J Int Neuropsychol Soc 2:441-451.

Mesulam M-M (1999) Spatial attention and neglect: parietal, frontal, and cingulate contributions to the mental representation and attentional targeting of salient extrapersonal events. Philos Trans R Soc Lond B Biol Sci 354:1325-1346.

Mort DJ, Malhotra P, Mannan S, Rorden C, Pambakian A, Kennard C, Husain M (2003) The anatomy of visual neglect. Brain 126:1986-1997.

Mort DJ, Malhotra P, Mannan SK, Pambakian A, Kennard C, Husain M (2004) Using SPM normalization for lesion analysis in spatial neglect. Brain 127:E11.

Nieuwenhuys R, Voogd J, van Huijzen C (1988) The human central nervous system: a synopsis and atlas. Berlin: Springer.

Ogden J (1985) Contralesional neglect of constructed visual images in right and left brain-damaged patients. Neuropsychologia 23:273-277.

Ota H, Fujii T, Suzuki K, Fukatsu R, Yamadori A (2001) Dissociation of body-centered and stimulus- centered representations in unilateral neglect. Neurology 57:2064-2069.

Rapcsak SZ, Watson RT, Heilman KM (1987) Hemispace-visual field interactions in visual extinction. J Neurol Neurosurg Psychiatry 50:1117-1124

Riestra AR, Crucian GP, Burks DW, Womack KB, Heilman KM (2001) Extinction, working memory, and line bisection in spatial neglect. Neurology $57: 147-149$.

Sakata H (2003) The role of the parietal cortex in grasping. In: Advances in neurology, Vol 93, The parietal lobes (Siegel AM, Andersen RA, Freund H-J, Spencer DD, eds), pp 121-139. London: Lippincott Williams and Wilkins.

Sereno MI, Pitzalis S, Martinez A (2001) Mapping of contralateral space in retinotopic coordinates by a parietal cortical area in humans. Science 294:1350-1354.

Shapiro K, Hillstrom AP, Husain M (2002) Control of visuotemporal attention by inferior parietal and superior temporal cortex. Curr Biol 12:1320-1325.

Stone SP, Halligan PW, Marshall JC, Greenwood RJ (1998) Unilateral neglect: a common but heterogeneous syndrome. Neurology 50:1902-1905.

Vallar G (2001) Extrapersonal visual unilateral spatial neglect and its neuroanatomy. NeuroImage 14:S52-S58.

Vallar G, Perani D (1986) The anatomy of unilateral neglect after righthemisphere stroke lesions. A clinical/CT-scan correlation study in man. Neuropsychologia 24:609-622.

Vallar G, Perani D, Cappa SF, Messa C, Lenzi GI, Fazio F (1988) Recovery of aphasia and neglect after subcortical stroke: neuropsychological and cerebral perfusion study. J Neurol Neurosurg Psychiatry 51:1269-1276.

Vallar G, Sandroni P, Rusconi ML, Barbieri S (1991) Hemianopia, hemianesthesia, and spatial neglect: a study with evoked potentials. Neurology 41:1918-1922.

Vallar G, Bottini G, Paulesu E (2003) Neglect syndromes: the role of the parietal cortex. In: Advances in neurology, Vol 93, The parietal lobes (Siegel AM, Andersen RA, Freund H-J, Spencer DD, eds), pp 219-315. London: Lippincott Williams and Wilkins.

Watson RT, Valenstein E, Day A, Heilman KM (1994) Posterior neocortical systems subserving awareness and neglect. Arch Neurol 51:1014-1021.

Young MP (1992) Objective analysis of the topological organization of the primate cortical visual system. Nature 369:448-450. 